\title{
Teorías subjetivas de profesores en reuniones de trabajo: un estudio descriptivo-interpretativo
}

\author{
Mariella Brighenti Bortoluzzi \\ Universidad de La Serena, Campus Andrés Bello, Chile \\ Jorge Catalán Ahumada \\ Universidad de La Serena, Campus Andrés Bello, Chile
}

\section{Resumen}

Este estudio es parte de un proyecto de desarrollo de la reflexión colectiva sistemática del profesorado, que cuenta con tres etapas: antes, durante y después de una intervención. Se reporta la fase inicial ("antes”), de observación y registro de reuniones de trabajo de profesores de un establecimiento educacional técnico-profesional de La Serena, Chile, durante cuatro meses, con el objetivo de investigar las Teorías Subjetivas que afloran en el quehacer docente, para acceder a cómo se plantean los profesores frente a sus problemas cotidianos y las posibles soluciones a ellos. A través de este análisis, fue posible teorizar sobre la importancia del rol docente y sobre cuáles serían los factores obstaculizadores y facilitadores del desarrollo profesional del profesorado.

Palabras Clave: Subjetividad, trabajo docente, desarrollo profesional.

\section{Subjective theories made by teachers in work meetings: a descriptive-interpretive study}

\begin{abstract}
This article is part of a development project of systematic collective reflection of the teachers, which has three stages: before, during and after an intervention. We report only the initial phase of observation and recording meetings of the teachers in La Serena. Observations with the aim to study the subjective theory of the problems that arise in the teaching work lasted three months. How the teachers pose in front of their everyday problems and possible solutions for them are discussed. Through this analysis it was possible to theorize about the importance of the role of teachers and what would be the factors hindering and facilitating professional development of teachers.
\end{abstract}

Keywords: Subjectivity, teaching work, professional development.

\section{Teorias subjetivas de professores em reuniões de trabalho: um estudo descritivo-interpretativo}

\section{Resumo}

Este estudo faz parte de um projeto de desenvolvimento da reflexão coletiva sistemática de professores que se constitui em três etapas: antes, durante e depois de uma intervenção. O artigo apresenta a fase inicial ("antes"), de observação e registro de reuniões de trabalho de professores de um estabelecimento educacional técnico-profissional de La Serena, Chile. Esta fase realizou-se durante quatro meses com o objetivo de pesquisar as Teorias Subjetivas que emergem no trabalho docente para conhecer como os professores se posicionam frente aos problemas cotidianos e às suas possíveis soluções. A partir da análise dos dados foi possível teorizar sobre a importância do papel docente e sobre quais seriam os fatores que dificultam e os fatores que facilitam o desenvolvimento profissional dos professores.

Palavras Chave: Subjetividade, trabalho docente, desenvolvimento profissional. 


\section{Introducción}

El presente artículo pretende aportar a la comprensión de cómo los profesores se posicionan frente a algunos fenómenos que ocurren en el sistema educativo. Estos fenómenos pueden ser entendidos como los problemas existentes en la escuela y las soluciones planteadas para ellos; las acciones planeadas, ejecutadas o no para sanarlos; y por ende, los fenómenos de orden interno- como las creencias, los pensamientos, las frustraciones y las perspectivas- de estos miembros de la comunidad escolar.

El estudio es un análisis de las creencias y opiniones, elaboradas como Teorías Subjetivasv- con lo cual se buscó comprender el pensamiento del profesorado, y reconocer los posibles factores facilitadores y obstaculizadores del desarrollo del profesor como profesional reflexivo, que actúa con racionalidad e intencionalidad.

Se realizaron observaciones de las reuniones de esos profesores, quienes expusieron sus experiencias acumuladas durante sus carreras docentes, sus sentimientos y posicionamientos frente a los problemas enfrentados en la escuela y sus sugerencias para enfrentar dichos problemas.

Para acceder a estos fenómenos e interpretarlos, trabajamos escuchando los relatos de los profesores en los que expresaban sus sentimientos, sus dificultades, sus estados de ánimo, frustraciones, pero también sus perspectivas de futuro y el posible logro de resultados positivos.

Para Shulman (conforme citado por Behrsin \& Selles, 2001 , p. 4) la profesión docente necesita de "memoria", pues "una de las frustraciones de la enseñanza como ocupación y profesión es su gran amnesia colectiva e individual". Se asume, entonces, que al traer sus experiencias al grupo, el profesor tiene la oportunidad de revisar su propia práctica y este ejercicio puede contribuir para "decodificar" el trabajo docente. El intercambio de vivencias y formulaciones teóricas es capaz de provocar el desarrollo de actitudes reflexivas.

Siendo así, en la construcción de nuestro objeto de estudio, la manera en que los profesores se entiendan a sí mismos en el ejercicio profesional puede ser muy importante para su desarrollo, pues tales impresiones están cargadas de sentidos propios y teorías subjetivas de cómo ser profesor. Además "para comprender la conducta profesional del profesor de una forma adecuada es necesario la realización de un análisis profundo de cómo se miran los profesores a sí mismos a lo largo de su carrera profesional" (Santaella, conforme citado por Behrsin \& Selles, 2001, p. 4).

La investigación, al mismo tiempo que intenta profundizar el conocimiento sobre la profesión docente, busca descubrir problemáticas involucradas en el desarrollo profesional del profesorado, identificar las teorías subjetivas que éstos formulan respecto a su práctica y descubrir de qué manera reflexionan estos profesionales sobre su rol de educador. Juzgamos importante debatir sobre la reflexión, pues se vincula estrechamente con la calidad educativa, ocupando un lugar central para promover el desarrollo profesional e indispensable para conferirle poder y autonomía al profesor (Catalán, 2010; Villar, 1988)
Basados en las consideraciones precedentes, intentamos responder a la siguiente pregunta:

\section{¿Cómo piensan y reflexionan los profesores cuando abordan colectivamente las problemáticas de su quehacer profesional?}

La aproximación teórica al fenómeno estudiado se realizó desde las Teorías Subjetivas, especialmente en aspectos relacionados con su conceptualización y el uso de ellas como fundamento a las acciones que las personas realizan.

\section{Teorías Subjetivas}

Las Teorías Subjetivas de Groeben y Scheele son una reinterpretación de los constructos personales de Kelly (conforme citado por Castro, 2008); sin embargo, la preferencia por atribuir el nombre "subjetivas" y no, "implícitas", se debe al carácter inclusivo de la teoría, pues esta nueva denominación permite considerar la posibilidad de estudiar tanto las teorías personales explícitas o declaradas por los sujetos, como las implícitas (Polanyi, conforme citado por Castro, 2008).

Según Groeben y Scheele, "las teorías subjetivas son cogniciones de la visión de sí mismo y del mundo, que se pueden entender como un conjunto complejo, que tiene una estructura argumentativa, por lo menos implícita, y que cumple funciones de explicación, predicción y tecnología, contenidas también en las teorías científicas" (Groeben \& Scheele, conforme citados por Avendaño, Krause, \& Winkler, 1993).

El concepto de TS suele utilizarse para hacer referencia exclusivamente al saber lego; sin embargo, existe una analogía entre el saber de sentido común y el saber de la ciencia. Algunos autores plantean que entre las teorías científicas y las del sentido común o intuitivas existen similitudes y diferencias, pero que unas y otras contienen elementos formales que justifican la denominación común de "teorías", pues de acuerdo con Rodrigo (1985), están constituidas por conceptos y por relaciones que se establecen entre éstos. Además comparten funciones interpretativas, pues a partir de los datos que obtienen, elaboran explicaciones basadas en postulados teóricos. En cuanto a sus funciones, las teorías subjetivas se corresponden a las teorías científicas, diferenciándose en que son más restringidas y porque no tienen pretensión de universalidad (Catalán, 2010).

Sistematizando el concepto de TS, se puede decir que las concepciones se construyen socialmente, pero funcionan individualmente en el mundo de cada individuo y no son, para quien las poseen, una abstracción o una convención, sino una forma de simplificar la realidad, conscientemente o no (Catalán, 1997).

\section{Objetivos}

Analizar los discursos de profesores de un establecimiento de educación media técnico profesional cuando 
abordan su propio quehacer, los problemas que enfrentan y las soluciones o explicaciones que encuentran para ellos, y conectando sus argumentaciones con el contexto en que surgen $_{\perp}$ caracterizar sus teorías subjetivas. Identificar los elementos facilitadores y obstaculizadores de la reflexión colectiva de los profesores, a través del análisis y síntesis interpretativa de los discursos de sus reuniones de trabajo.

\section{Diseño Metodológico}

El presente trabajo trata de un estudio de caso de carácter descriptivo-interpretativo. Se recurrió a la metodología cualitativa, que incluyó la observación exploratoria para la recolección de los datos, siendo una investigación empírica, intensiva y en profundidad de los fenómenos que ocurren naturalmente en el ambiente de trabajo de un grupo de profesores. La unidad de análisis estuvo compuesta por 33 profesores y profesoras de un establecimiento de administración municipal de educación técnica de la ciudad de La Serena- Chile.

\section{Procedimientos Empleados para la Recolección de Ios Datos}

La recolección de datos se llevó a cabo en un período de cuatro meses. Consistió en observaciones con registros escritos detallados, grabación en audio y videos de diez sesiones de trabajo del grupo de profesores estudiado.

En esta etapa de la investigación, todo lo expresado verbalmente por los profesores fue transcrito. Se buscaba identificar elementos que pudiesen constituir Teorías Subjetivas, sin embargo la comprobación de que los textos correspondientes pudieran ser considerados TS solamente vendría después de un análisis más profundo y de su comprobación con la ayuda del material recolectado en audio, además del trabajo de triangulación con investigadores externos.

\section{Resultados}

Para fines de interpretación, este reporte contará con dos momentos de análisis. Esta división se hizo a partir de la consideración de que el proceso seguido por los profesores para abordar los problemas permite identificar dos momentos, íntimamente relacionados. Conforme veremos en la secuencia, el primer momento puede ser entendido a partir de dos perspectivas: como un diagnóstico de los problemas enfrentados en la escuela o, como plantea Catalán (2010), un agente inhibidor de acciones. Así, "las teorías inhibidoras de acción son teorías que eximen a quien las sustenta de la responsabilidad de realizar acciones, en virtud de que pudieran resultar poco deseables, inconducentes, difíciles o atentatorias contra su seguridad. Son argumentos para no realizar ciertas acciones" (p. 76).
El segundo momento puede ser entendido como una fase más reflexiva en la búsqueda de soluciones.

\section{Momento Uno}

Las dos primeras reuniones observadas dan cuenta de un diálogo entre el profesor responsable de cada curso y los miembros del cuerpo directivo. La pauta de reunión incluía la inasistencia, el mal comportamiento y el bajo rendimiento. Los profesores discuten los problemas de las alumnas y sus actitudes en aula. Se refieren a las alumnas que actúan de manera no deseada y apuntan cuál sería el motivo para dicho comportamiento.

La dinámica observada de la reunión puede ser descrita como una sesión de preguntas y respuestas. El director (en algunos casos era la Jefe de Unidad Técnico Pedagógica) preguntaba a los profesores cuáles son las alumnas que presentaban los problemas mencionados y el profesor responsable contestaba, dando el nombre de la alumna y la posible causa para el comportamiento. Se percibe que la misma causa es mencionada innumerables veces, para distintas alumnas. Llamó la atención del investigador la recurrencia de una frase dicha por los profesores cuando se habla de comportamiento en general: "esta niña tiene problemas familiares, entonces presenta mal comportamiento".

Se puede inferir con facilidad que los profesores saben lo que pasa con sus alumnas en la escuela y fuera de ella, conviven con ellas y observan sus conductas, lo que les permite hacer juicios y consideraciones, como afirmar que algunas son "indolentes, prejuiciosas y prepotentes":

"ella tiene una visión de mundo de que el mundo está equivocado y ella no, entonces actúa como le conviene" (sesión 2)

"ella busca autoafirmación" (sesión 2)

Se percibió claramente que la relación que los profesores mantienen con las jóvenes es estrecha. Es decir, las conocen bien, conocen sus familias y hablan constantemente con los padres, además saben lo que les pasa y las relaciones que mantienen entre ellas fuera de la escuela y que afectan directamente la convivencia en el ambiente escolar, sus problemas y situaciones perturbadoras que, según los profesores, acaban por interferir en el desarrollo de las clases.

"las alumnas tienen problemas entre ellas...la rivalidad entre ellas pasa también en la calle" (sesión 5)

"los conflictos entre las alumnas son externos...novios, tribus urbanas" (Sesión 5)

"las alumnas discriminan alguien diferente, una chica que es homosexual...otra que hace el género buenamoza" (sesión 5). Además, se desprende de las citas que hay 
intentos de comprender los antecedentes que llevan al mal comportamiento de las estudiantes y que hacen atribuciones causales acerca de ellas y del buen desarrollo de las clases.

"las niñas tienen mal comportamiento porque siguen a líderes negativos" (sesión 2)

"esta niña no tiene control sobre sus emociones, entonces presenta problemas de conducta" (sesión 2)

"la actitud de ella tiene mucho que ver con la manera que ella enfrenta sus problemas personales" (sesión 2)

"ella pasa por problemas que son de gente mayor" (sesión 2)

"las niñas necesitan un líder, ven al profesor como un padre" (sesión 5)

Las carencias de las alumnas y sus problemas emocionales son frecuentemente mencionados para explicar o justificar las cuestiones claves discutidas en las reuniones. El cuerpo directivo y el cuerpo pedagógico constantemente presentan posibles motivos para las actitudes no deseadas que interfieren en el buen funcionamiento de la escuela.

Llama la atención el hecho de que para todos los problemas citados anteriormente y otros, como falta de motivación y falta de interés de las alumnas -motivos que conducen al bajo rendimiento- son entendidos por los profesores como cuestiones ajenas a ellos.

Creen que los problemas económicos y la cultura familiar son los principales factores causantes. Atribuyen la responsabilidad a las fallas de la educación, a factores externos, variables no manipulables e inmutables.

Cuando se trata del bajo rendimiento, los profesores frecuentemente dicen que una u otra alumna "tiene problemas de aprendizaje". Esto significa que el profesor considera que su estudiante tiene un déficit y debe someterse a un programa especial de aprendizaje, y a partir de este momento ya no es más su responsabilidad.

Ya en los casos en que se descarta la posibilidad de déficit, los profesores tienen otras explicaciones que justifican el bajo rendimiento de las alumnas.

"las alumnas tienen bajo rendimiento por falta de interés" (sesión 1)

"tienen la autoestima muy baja y eso lleva a no esforzarse y no motivarse" (sesión 5)

"yo pienso que en cuanto a los compromisos las niñas son muy relajadas"(sesión 2)

"uno tiene que preparar la prueba ahí, o la prueba queda en blanco, jes flojera!"(sesión 2)

"el rendimiento es bajo porque falta el esfuerzo de la familia, la familia es fundamental" (sesión 1)
Además de estos factores, como se puede apreciar en la última cita, los profesores juzgan la participación de la familia como principal responsable por el buen desarrollo de la educación en general. Mencionan reiteradamente la importancia de la participación de los padres en el rendimiento escolar, inasistencia y mal comportamiento y comentan la falta de interés de éstos en las cuestiones del aprendizaje de sus hijas. Como vemos en: "el último SIMCE obtuvo buenos resultados, pero los apoderados no quisieron ni saber" (sesión 3).

En el intento de explicar lo que ocurre en el ambiente escolar, los profesores hacen referencia a lo que llaman problemas sociales -de orden económico- y los problemas personales, que tienen directamente que ver con la institución familiar y los déficits de ésta en la educación moral, sentimental y psicológica de las niñas.

"ella es una chica con muchos problemas sociales... familiares y falta de afecto" (sesión 2)

"la alumna quiso suicidarse, porque ya no tenía ganas de vivir. La mamá hace de cuenta que ella no existe y ella hace de cuenta que no tiene mamá" (sesión 2)

"las alumnas vienen de hogares deficientes y traen para las clases sus problemas personales" (sesión 5)

"en sus casas las alumnas no están en un ambiente propicio para aprender valores y los aprenden aquí en la escuela" (sesión 5)

Frente a situaciones como las expuestas en las citas precedentes, queda en evidencia que los profesores atribuyen a factores externos gran parte de los problemas enfrentados en la escuela y, por lo tanto, sus soluciones no dependen de su intervención. Se puede advertir que en algunos momentos hay el sentimiento o la voluntad de deshacerse de los problemas de las alumnas, enviándolas a otro establecimiento o insertándolas en programas especiales 0 , simplemente, manteniendo la situación como está.

"tenía todas las calificaciones bajas, no podemos hacer nada más por ella" (sesión 1)

"considero que hay niñas que no cumplen el perfil para ejercer esa carrera” (sesión 8)

"se entrega demasiada información, es material perdido" (sesión 5)

"las niñas que no leen comprensivamente nunca van aprender como se espera" (sesión 9).

Con lo que vimos hasta ahora y las citas que continúan, planteamos la existencia de una situación que se puede interpretar como de conformidad o de resignación. Dicha conformidad puede ser sustentada a modo de teorías subjetivas identificadas como mantenedoras de acción, "que 
tienden a sustentar acciones que se realizan en forma recurrente o habitual" (Catalán, 2010, p. 76), o aún como teorías subjetivas inhibidoras de acción.

\section{Actividad reflexiva propuesta por el directorio del liceo}

El directorio del liceo presentó a los profesores un ejercicio de reflexión y auto evaluación a través de un cuestionario.

Una de las citas que llamó la atención del investigador fue que al preguntar sobre el planeamiento de las clases, hubo estas respuestas:

"no están preguntando si resulta, pero si se hace... y sí, se hace”(sesión 3)

"cuando uno planifica sale precioso, pero en la práctica..." (sesión 3))

"yo no planeo mis clases, tengo un libro de donde las saco listas" (sesión 3)

La primera cita una vez más ratifica una situación de conformidad. Es decir, no importa que no haga bien el trabajo, mientras lo haga. La segunda cita viene a ser la constatación de "trabajo perdido", motivo que justificaría mantener una oposición a planificar las clases.

En las citas siguientes aparece más claramente la conformidad en relación a los contenidos de las clases. Se preguntó si los profesores planean las clases de manera que el contenido constituya un desafío, garantizando el interés y la motivación de las alumnas.

"para que el contenido fuera desafiador para las alumnas, yo tendría que tener un laboratorio experimental” (Sección 3)

"en mi caso, tendrían que tener conocimiento general, no saben de la guerra del Irak, ni del nombre del río por el que pasan al lado todos los días" (sesión 3)

"ellas tienen un bajo nivel de conocimiento, por eso el contenido es desafiador" (sesión 3)

Cuando se pregunta en el cuestionario cuáles son las estrategias de enseñanza para alcanzar los objetivos, algunas de las respuestas fueron:

"se logra maquiavélicamente" (sesión 3)

"uno hace un paralelo del contenido, lo acerca al mundo" (sesión 3)

"no importa si preguntan tonterías, lo peor es cuando no preguntan nada"(sesión 3)
Aparte del uso del miedo de las calificaciones, se esboza un cierto nivel de compromiso por parte de los profesores. Hay esfuerzo también, y por más que muchas veces sus palabras reflejen superficialidad de ideas, o puramente intentos defensivos, existen planteamientos - ya en este momento - que muestran voluntad de asumir más responsablemente los problemas.

Vimos, con los ejemplos de TS presentados y discutidos hasta aquí, que hay situaciones en las cuales las respuestas de los profesores pueden ser entendidas como excusas para eximirse de la responsabilidad por los problemas enfrentados en su quehacer diario. Sin embargo, también vimos que hay otras situaciones que pueden ser entendidas simplemente como una elaboración del diagnóstico de estos problemas, sin por lo tanto la carga de conformidad recurrente.

Según los registros, al parecer, las jóvenes no tienen muchos problemas en las empresas en que trabajan, ejerciendo las funciones que se les enseñan en la escuela. Esto puede llevarnos a pensar que quizás las clases no les sean interesantes, por factores pedagógicos o de estrategias de enseñanza y éstos puedan ser factores relevantes en los problemas de bajo rendimiento e inasistencia. No obstante, estas posibles causas no fueron en ninguna situación mencionados por los profesores, pues siempre atribuyeron las causas de los problemas a factores externos.

"yo pienso que las niñas se contradicen, porque faltan a las clases por problemas personales, pero no faltan al trabajo en la empresa" (sesión 10))

"¿qué hacer para que las alumnas tengan en la escuela el mismo desempeño que tienen en la empresa? Hay que buscar que está pasando, hay que buscar otras variables que manejar" (sesión 10)

\section{Momento Dos}

En este momento, se puede observar que el discurso de los profesores ya no refleja la pura descripción de los problemas inherentes a la escuela, sino las ideas o aportes de estos profesores para llegar a un acuerdo y resolver dichos problemas.

Entre las soluciones propuestas para resolver los problemas, comúnmente relatados por los profesores, está el intento de crear un ambiente favorable al aprendizaje. Los ítems que consideran importantes para que se cree dicho ambiente son:

1. Adoptar actitudes contra los estímulos distractores de la atención en el aula: "Creo que si los profesores contestan sus celulares las alumnas también creen que pueden, por eso tenemos que darles el ejemplo" (sesión 4)

2. Mejorar las relaciones interpersonales, a través del diálogo: "hay que escucharlas, tiene que haber 
diálogo, comunicación, para crear un vínculo y mejorar el ambiente de clase" (sesión 5)

"tenemos que saber los problemas socio afectivos de las alumnas para lidiar mejor con ellas, si no sabemos lo que pasa con ellas no podemos ayudar" (sesión 5)

También está presente la mutua relación alumno/profesor/alumno. Además, se puede inferir disposición a intervenir, lo que se revelaría en la aparición de teorías subjetivas iniciadoras de acción (Catalán, 2010, p. 76).

"quedo 14 horas y sé lo que piensan y sienten las chicas, nosotros somos llevados como ejemplo" (sesión 5)

"hay niñas enfermas (sobre una niña que lloraba en la clase) y ellas también necesitan tratamiento" (sesión 5)

3. Diálogo entre los miembros del grupo para solucionar problemas colectivamente.

"nosotros pensamos que somos súper y no nos interesamos por nuestra situación personal...como profesores que tienen problemas personales o están enfermos y podrían compartir con el colega y eso ayudaría en el ambiente favorable" (sesión 5)

"la disciplina y la convivencia es responsabilidad de todos... en conjunto" (sesión 6)

4. Existe la preocupación por la deserción escolar. Se puede reconocer una vez más la presencia de teorías subjetivas iniciadoras de acción.

"de nosotros dependerá que las niñas siegan en la escuela hasta el cuarto medio"

"si la niña se siente incómoda en un curso, hay que cambiarla" (sesión 6)

"cuidemos las chiquillas, para que no se pongan a perder" (sesión 6)

5. Aunque tímidamente, existe la autocrítica.

"uno tiene que reconocer y auto-evaluarse" (sesión 3)

"nosotros somos productos de todos los profesores que nos aportaran algo, así que..."(sesión 9)

Un aspecto destacable, que sin constituir teorías subjetivas, puede tener incidencia en la adopción de una actitud propositiva de los docentes es la presencia de incentivo, de refuerzo positivo, por parte de la directora de la escuela hacia los profesores. Las citas que se encuentran abajo tienen la directora como sujeto hablante. "todo lo que logramos fue por ustedes que hacen la parte gruesa del trabajo"

"les agradezco su comprensión y disposición en incluir a una alumna trasplantada"

"las niñas vienen con su uniforme completo, están bien presentadas, les felicito"

\section{Discusión}

Esta investigación es puramente observacional, debido a su subordinación a un proyecto más grande, que incluye una primera fase sin intervención de los investigadores. Siendo así, el trabajo tiene sus límites teóricos y prácticos, relacionados con el acceso a los procesos reflexivos de los profesores, a pesar de lo cual ha permitido describir e interpretar explicaciones que los profesores sustentan cuando abordan problemáticas de su quehacer cotidiano en sus reuniones de trabajo.

\section{¿Excusas o Diagnóstico?}

Se plantea la hipótesis que el discurso de los profesores refleja que éstos tienen un diagnóstico institucional, basado en sus observaciones y la convivencia diaria. Este diagnóstico se manifiesta a modo de teorías subjetivas, por su carácter argumentativo (se expresa como una consecuencia a partir de un antecedente). Estas teorías tienen una estructura superficial, pues se accede a ellas oyendo directamente lo verbalizado por los profesores. También tienen una estructura profunda (Catalán, 2010) subyacente que hace que diversos enunciados correspondan a una misma explicación (como por ejemplo, las explicaciones acerca del mal rendimiento escolar tratadas anteriormente)

Se percibe con posterioridad a la elaboración del diagnóstico, que los profesores encuentran soluciones para los problemas relatados, aunque éstas sean meramente teóricas o discursivas. En un segundo caso, las verbalizaciones de los profesores plantean excusas o externalizan los factores causales de los problemas. Podemos decir que las atribuciones de los profesores, corresponden a un locus de control externo, es decir, sus argumentos les liberan de responsabilidad.

El control externo se expresa de forma independiente del propio comportamiento de la persona o sus características personales. Es decir, la persona espera que la información o resultado sea una función del azar, suerte o destino, bajo el control de otros poderes, o simplemente impredecible (Rotter, 1966). Este hecho es un fuerte indicio de que las teorías subjetivas sustentadas, reconocibles por su orientación a la acción como mantenedoras o inhibidoras de acción, son factores obstaculizadores de la reflexión y del desarrollo profesional del profesor (Catalán, 2010, 2011). 


\section{¿Excusas o respuesta inconsciente?}

Podemos aún intentar encontrar un origen o un porqué para la formulación o sustentación de las Teorías Subjetivas Inhibidoras y Mantenedoras de acción por parte del profesorado. Se puede decir que el acto de delegar causas a factores externos es una manifestación de la presencia de teorías subjetivas de alto compromiso emocional (Catalán, 2010) por su fuerza argumentativa, en defensa propia, frente a una situación incómoda o quizás una exigencia por resultados o respuestas, y que esta acción de defensa se debe al hecho de que la exigencia por la calidad de la educación viene desde adentro de la escuela como igualmente viene desde afuera, por parte de la sociedad. Por una parte, la aparición de este tipo de teorías subjetivas obedecería a una búsqueda intencionada de excusa o justificación; sin embargo, por otra parte, también hay indicios de que su aparición ocurra de manera más o menos inadvertida por los propios docentes, cual si ante ciertas demandas emergentes surgieran como respuesta casi instintiva (poco consciente) o automática (mecánica), originada en una estructura más profunda, implícita o invisible, que puede ser inferida o reconstruida total o parcialmente.

Podemos intentar explicar ese fenómeno a través de la teoría de los constructos personales de Kelly (conforme citado por Castro, 2008) que consiste en la formulación de un sistema teórico acerca de la realidad, es decir "un sistema individual de construcción del medio" (Kelly, conforme citado por Castro, 2008, p. 43). Dicha construcción estaría compuesta por conceptos o constructos bipolares con organización jerárquica que actuarían como ejes de referencia en la interpretación de la realidad (Ávia, conforme citado por Castro, 2008). Esa jerarquía puede ser explicada en la teoría del núcleo central de las representaciones sociales, que plantea que "no todo constructo personal es igualmente susceptible de ser modificado mediante la experiencia, lo cual dice relación con la diferencia que Kelly establece entre construcciones personales nucleares y periféricas" Castro (2008, p. 44).

Así, los constructos nucleares podrían ser entendidos como siendo los aspectos centrales del pensamiento, que poseen mejor capacidad y más posibilidades de aplicación "teórica, en el sentido subjetivo, y por tanto más jerarquía" (Kelly, Pervin, conforme citado por Castro, 2008, p. 44). Esa clase de constructos serían más profundos y resistentes al cambio y tienen "como misión gobernar los procesos de mantenimiento personales" (Kelly, conforme citado por Castro, 2008, p. 45).

Hay registros de muchas respuestas rápidas de los profesores, como por ejemplo, ante preguntas relacionadas con el comportamiento y el rendimiento de las alumnas. Se trata de respuestas que podrían considerarse automáticas, más cercanas a un acto reflejo que a un acto reflexivo. En lo referente a la velocidad de las respuestas, fue posible percibir que éstas tienen carácter anticipatorio o predictor, lo que evidencia otra característica importante de la teoría de los constructos personales de Kelly (conforme citado por
Castro, 2008) y más tarde planteado por Ávia(1994) que describe que " todos los procesos psicológicos de una persona, incluida su conducta externa, están determinados por el modo en que un individuo anticipa lo que va a pasar en el futuro..." (conforme citado por Castro, 2008, p. 43).

\section{Manifestación de la Proactividad}

Los argumentos orientados a la realización de acciones, el empeño en solucionar problemas, son considerados factores facilitadores del cambio comportamental, del pensamiento crítico y la conciencia, que conllevan al desarrollo profesional. Esta manifestación ocurre inicialmente en forma de Teorías Subjetivas Iniciadoras de Acción (Catalán, 2010)

Se evidencia en lo comunicado por los profesores que después de una elaboración de diagnóstico institucional o de plantear causas que atribuyen a factores externos, ellos elaboran, buscan, o imaginan soluciones teóricas para los problemas, sin importar cuál sea su naturaleza. En dichas soluciones pensadas y comunicadas por los profesores, se comprueba que hay la intención de actuar, de realizar otras acciones para encontrar otros resultados.

Sin embargo, no podemos afirmar que el hecho de que el profesor exponga una propuesta de cambio, garantice que esta reacción proactiva pasará del campo de las teorías subjetivas al ejercicio de su rol profesional. Existen variables organizacionales que obstaculizan los emprendimientos de esos profesionales, como la falta de autonomía, y las reglas o leyes de la institución que limitan la creatividad. Gore plantea que los problemas de los profesores no son problemas personales, sino organizativos "Una organización es un sistema de vínculos. Rara vez la calidad de la educación en una escuela puede ir más allá de la calidad de estos vínculos" (Gore, 1996, p. 303)

\section{El círculo vicioso}

Con fines interpretativos del estudio que se reporta, planteamos la hipótesis de que un posible círculo vicioso se produce en las reuniones de este grupo de profesores. El ciclo se iniciaría con la fase de diagnóstico, en la que los profesores reconocen los problemas que existen en la escuela y sus causas, que delegan generalmente a factores externos.

Pasaría a una segunda fase, en la que los profesores plantean soluciones teóricas para dichos problemas, muchas veces con teorías subjetivas iniciadoras de acción, es decir, con argumentaciones que impelen a la acción.

En la secuencia, el círculo pasa por la fase de la acción o de la no-acción. En caso de ser efectiva la acción por parte de los profesores, ésta tendrá resultados positivos o negativos. En caso de la no-acción, seguirán los mismos problemas u otros sumados a ellos. Por cierto, la acción no es reflejo fiel del pensamiento. La relación entre teorías sustentadas y la acción no es directa ni inmediata (Kolbe \& Boos, 2009). 
El resultado es que volveríamos a la fase inicial, en la cual los profesores relatan los problemas una vez más.

Otro factor que refuerza la hipótesis de dicho ciclo, es la cantidad de reuniones semanales sumadas a los muchos años de la carrera en la escuela. Los profesores ya conocen hace mucho los problemas que afectan a la escuela y son constantemente cuestionados sobre ellos. Podría entonces estar actuando en algunos casos:

- Un mecanismo más reflejo que reflexivo: de defensa propia delegando causas de problemas a factores externos.

- Las llamadas rutinas docentes: "surgidas de la propia experiencia y que se convierten en criterios de validez para la toma de decisiones. Su importancia radica en que permanecen como fundamento oculto a la conciencia de los profesores, incluso en los expertos, constituyendo sus ideas implícitas que, al ser usadas como referentes, les produce conductas muy seguras y resistentes al cambio". (De Longhi, conforme citado por Jiménez \& Wamba, 2003, p. 115). "La experiencia tiene límites, después de los cuales se convierte en rutina". (Gore 1996, p. 304).

\section{Conclusión}

Los profesores observados en este estudio, cuando abordan problemáticas de su quehacer en reuniones de trabajo, tienden a desarrollar un proceso con dos fases claramente diferenciadas, que hemos denominado "momentos reflexivos". Si bien en ambos se realiza análisis y alguna forma de reflexividad, hay entre ellos una notoria diferencia en la profundidad, como en la responsabilidad para apropiarse de los problemas.

El primer momento se caracteriza por un nivel de reflexión elemental, automático y casi instintivo, que puede ser más cercano a lo inconsciente que a un nivel reflexivo. Este es uno de los factores obstaculizadores del desarrollo profesional encontrados en esta investigación.

En un segundo momento, se percibe que tienen un ejercicio de reflexión y progresiva conciencia de que sí pueden manejar o controlar la situación. Lo que se caracteriza como un factor facilitador de cambios de teorías subjetivas, así como de la orientación a la acción de éstas.

Sin embargo, las soluciones que proponen no son innovadoras y el despliegue de la creatividad para generar propuestas está potencialmente disminuida, o restringida, por ser la escuela una organización, con sus reglas y límites.

Cabría entonces, explorar condiciones de gestión facilitadoras de procesos reflexivos colectivos de los profesores, que les permitieran incrementar la conciencia, asumir sus propias teorías, revisarlas y tener disposición a modificarlas, relacionarlas con sus actuaciones y ejercer genuinamente su autonomía profesional.
La idea de explicitar lo implícito, a través de la verbalización, se funda en la posibilidad de acceder a las TS más profundas y tomar conciencia de ellas mediante la reflexión.

Así, una forma de cambiar las teorías subjetivas mantenedoras e inhibidoras de acciones, sería el establecimiento de contactos sociales, que podrían influir en las argumentaciones de quienes las sustentan, tanto en relación a los contenidos al que se refieren, cuanto a la reconstrucción social de la representación personal (Rodríguez, Rodrigo, \& Marrero, 1993).

Por su parte, la noción de avance hacia la estabilidad de nuevas representaciones, reevaluadas mediante la reflexión o intervención de expertos, considera la construcción de nuevas concepciones o teorías, las que mediante el uso alcanzarían la estabilidad y por tanto, podrían desplazar a la teoría original (Pozo, 1999). Sería, entonces, conveniente realizar nuevas investigaciones que permitan dar cuenta de los cambios representacionales y las condiciones para producirlos.

\section{Referencias}

Avendaño, C., Krause, M., \& Winkler, M. I. (1993). Representaciones sociales y teorías subjetivas: relevancia teórica y aplicaciones empíricas. Psykhe, 2(1), 107-114.

Behrsin, M., \& Selles, S. E. (2001). Formação docente: análise de reflexões de professores de ciências sobre sua trajetória de desenvolvimento profissional. Recuperado: 01 mar 2014. Disponível: $\quad$ www.anped.org.br/reunioes/25/excedentes25/ mariacristinabehrsint08.rtf

Castro, P. J. (2008). Cambio de teorías subjetivas de profesores respecto a la enseñanza y el aprendizaje de valores. Tesis presentada para optar al grado de Doctor en Psicología, Facultad de Ciencias Sociales, Pontificia Universidad Católica de Chile, Santiago, Chile.

Catalán, J. (1997). La profesión del profesor según las teorías subjetivas de los formadores. Tesis de Doctorado para la obtención del título de Doctor en Ciencias de la Educación, Facultad de Educación, Pontificia Universidad Católica de Chile, Santiago, Chile.

Catalán, J. (2010). Teorías Subjetivas: Aspectos teóricos y prácticos. La Serena, Chile: Editorial Universidad de La Serena.

Catalán, J. (2011) Del pensamiento al conocimiento profesional del profesor. Em J. Catalán (Ed.). Psicología Educacional. Rumbos, problemáticas y aportaciones (pp. 187-215). La Serena: Editorial Universidad de La Serena.

Gore, J. (1996). Controversias entre las pedagogías. Madrid: Morata.

Jiménez, R., \& Wamba, A. M. (2003). ¿Es posible el cambio en los modelos didácticos personales?: obstáculos en profesores 
de ciencias naturales de educación secundaria. Revista interuniversitaria de Formación de profesorado, 17(01), 113-131

Kolbe, M., \& Boos, M. (2009). Facilitating Group Decision-Making: Facilitator's Subjective Theories on Group Coordination. Recuperado: 01 abr 2009. Disponível: http://www.qualitativeresearch.net/index.php/fqs/article/view/1244/2692

Pozo, J. I. (1999). Más allá del cambio conceptual: el aprendizaje de la ciencia como cambio representacional. Enseñanza de las Ciencias, 17(3), 513-520.

Rodrigo, M. J. (1985). Las teorías implícitas en el conocimiento social. Infancia y Aprendizaje, 31-32, 145-156.
Rodríguez, A., Rodrigo, M. J., \& Marrero, J. (1993). El proceso de construcción del conocimiento. Teorías implícitas o teorías científicas. Em M. J. Rodrigo, A. Rodríguez \& J. Marrero (Eds.), Las teorías implícitas. Una aproximación al conocimiento cotidiano. Madrid: Aprendizaje Visor.

Rotter, J. B. (1966). Generalized expectancies of internal versus external control of reinforcements. Psychological Monographs 80(609).

Villar, L. (1988), Conocimiento, creencias y teorías de los profesores. Implicaciones para el curriculum y la formación del profesorado. Madrid: Marfil.
Recebido em: 13/11/2012

Reformulado em: $13 / 07 / 2013$

Aprovado em: 23/07/2013

\section{Sobre os autores}

Mariella Brighenti Bortoluzzi (maribortoluzzi@gmail.com)

Mestranda, Psicología de la educación, Universidad de La Serena. En Chile

Jorge Catalán Ahumada (jcatalan@userena.cl)

Vicerrectoría académica, Universidad de La Serena, Campus Andrés Bello, Avenida Raúl Bitrán Nachary S/N

Este artigo é inédito. Foi derivado de uma dissertação de mestrado. Quando ainda em fase de conclusão, foi apresentado oralmente na IX Jornada Internacional de Psicología Educacional: Educandos, Formadores, Ciudadanos: Entrecruces para repensar la Educación.ValparaísoChile 2009.

Contou com o apoio de um grupo de discução para a triangulação dos dados, composto por: Castro, Pablo ; General, Francis ; Jofré, Richard ; Saez, Nicolás ; Vega, Angela.

O projeto foi financiado por DIULS/ Universidad de La Serena. 